\title{
Supporting Information \\ For \\ Coprecipitation of Heavy Metals in Calcium Carbonate from Coal Fly Ash Leachate
}

Heather A. Hunter, Florence T. Ling and Catherine A. Peters*

- AUTHOR INFORMATION

*Corresponding Author

Catherine A. Peters - Department of Civil and Environmental Engineering, Princeton

University, Princeton, New Jersey 08544, United States;

orcid.org/0000-0003-2418-795X;

Phone: (609)258-5645;

Email: cap@princeton.edu

Authors

Heather A. Hunter - Department of Civil and Environmental Engineering, Princeton University, Princeton, New Jersey 08544, United States; orcid.org/ 0000-0002-4692-1548

Florence T. Ling -- Department of Chemistry, La Salle University, Philadelphia, Pennsylvania, 19142, United States; orcid.org/0000-0002-2576-3608;

Contents:

Figure S1 Page S2

SEM image of the particle featured from the FAL experiment which is shown in the nano-XRF images in Figure 1 and in Figure S2a. The particle is on the Si mounting chip near Pt fiducial gridlines.

Figure S2

Page S3

Nano-XRF elemental maps showing distribution of calcium in a) the particle from the FAL experiment (the same particle shown in Figure 1) and b) the particle from the FAL_Fe experiment (the same particle shown in Figure 2). 


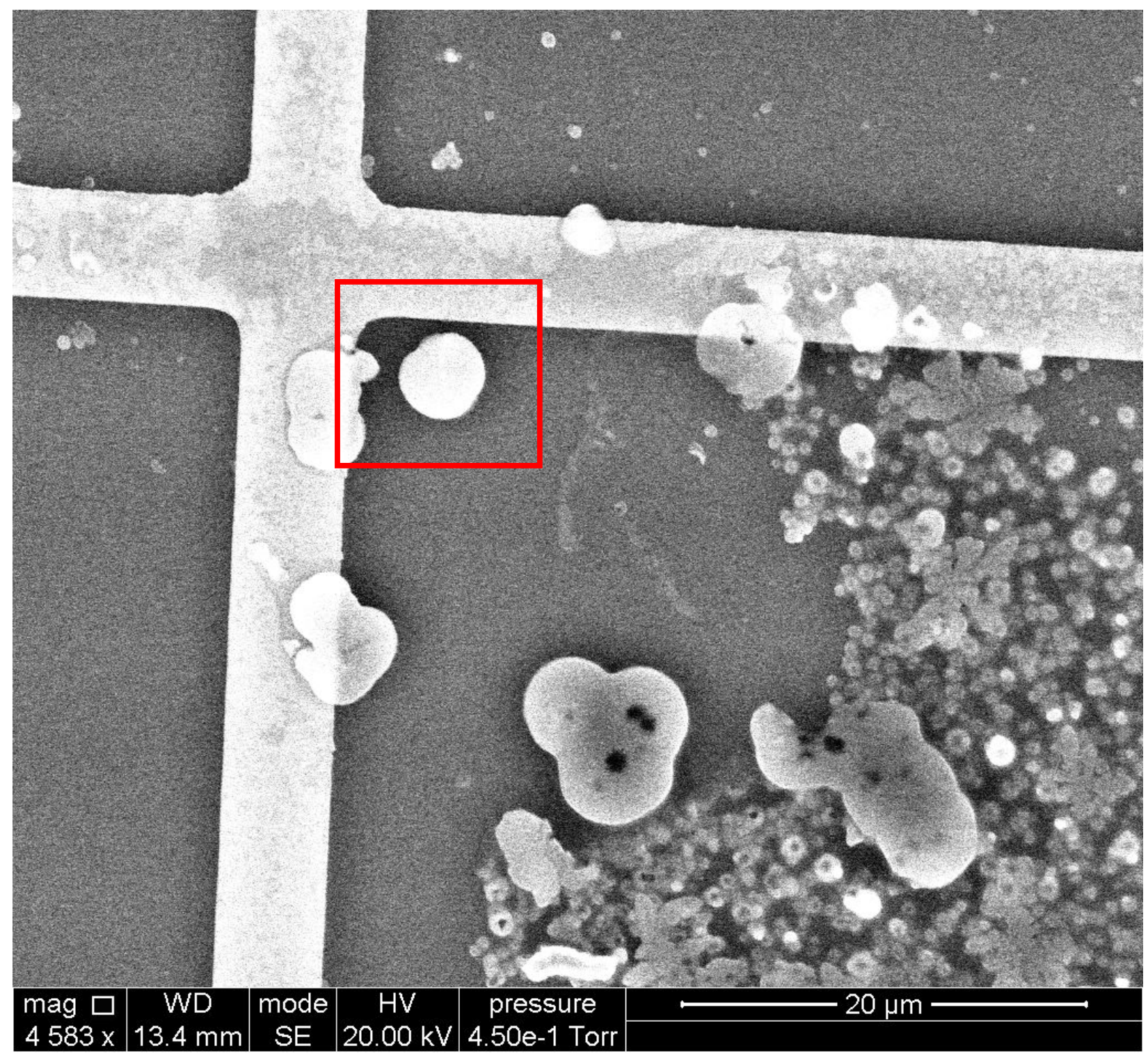

Figure S2. SEM image of the particle featured from the FAL experiment which is shown in the nano-XRF images in Figure 1 and in Figure S2a. The particle is on the Si mounting chip near Pt fiducial gridlines. 

(a) FAL
ppm Ca

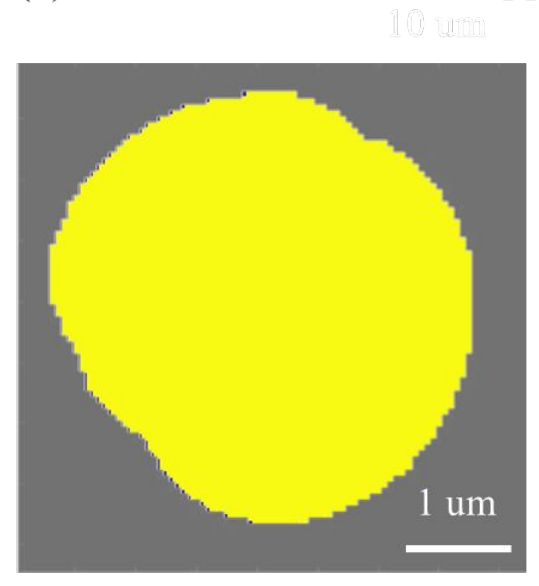

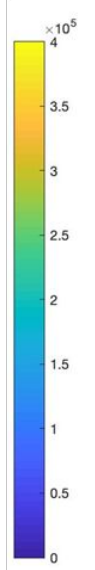

(b) FAL_Fe

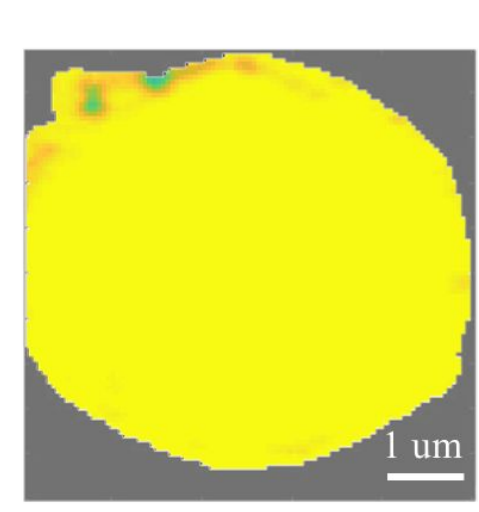

ppm Ca

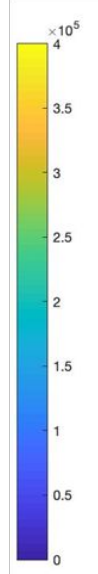

Figure S2. Nano-XRF elemental maps showing distribution of calcium in a) the particle from the FAL experiment (the same particle shown in Figure 1) and b) the particle from the FAL_Fe experiment (the same particle shown in Figure 2). 